Check for updates

Cite this: RSC Adv., 2019, 9, 4422

\title{
Synthesis of ZnO doped high valence S element and study of photogenerated charges properties $\uparrow$
}

\author{
Lijing Zhang, (D) Xiufang Zhu, Zhihui Wang, Shan Yun, (D) Tan Guo, Jiadong Zhang, \\ Tao Hu, Jinlong Jiang and Jing Chen
}

Nonmetal doping is an efficient way to increase the photoresponse range of $\mathrm{ZnO}$. However, the mechanism for improving the light response range of $\mathrm{ZnO}$ with nonmetal doping is not clear. Herein, $\mathrm{ZnO}$ doped with $\mathrm{S}$ was successfully prepared by ion exchange and calcination methods, which resulted in the uniform distribution of sulfur ions in $\mathrm{ZnO}$. The $\mathrm{S}$ element doped was mainly $\mathrm{S}^{4+}$ and $\mathrm{S}^{6+}$, which was identified by XPS. We studied the influence of $S$ on the photogenerated charge characteristics of ZnO with SPS. Results indicated that the uniform distribution of $S$ dopants elevated the valence band maximum by mixing S $3 p$ with the upper valence band states of $\mathrm{ZnO}$. The valence band maxima of $\mathrm{S}-\mathrm{ZnO}$ was $0.37 \mathrm{eV}$ higher than that of $\mathrm{ZnO}$. This result was the main reason for the improvement in the light response. We also studied the photocatalytic activity of $\mathrm{Ag} / \mathrm{S}-\mathrm{ZnO}$. Ag/S-ZnO with $10 \mathrm{wt} \% \mathrm{Ag}$ loading showed the highest photocatalytic degradation rate for $\mathrm{MO}$. In this paper, a potential photocatalytic mechanism has been proposed.

Received 18th September 2018 Accepted 24th December 2018

DOI: $10.1039 / \mathrm{c} 8 \mathrm{ra} 07751 \mathrm{~g}$

rsc.li/rsc-advances energy level impurities, which can enhance the visible-light response of $\mathrm{ZnO}$, improve carrier mobility rate and enhance the photogenerated charge separation efficiency.,23 For example, Feng et al. prepared sulfur-doped carbon nitride, which showed a higher photocatalytic $\mathrm{H}_{2}$ evolution activity than that by carbon nitride. ${ }^{24}$ Xiang et al. synthesized nitrogen and sulfur co-doped $\mathrm{TiO}_{2}$, which showed an enhanced photocatalytic activity for degrading 4-chlorophenol compared to pure $\mathrm{TiO}_{2} \cdot{ }^{25}$ However, there are relatively few studies on $\mathrm{S}$ doped $\mathrm{ZnO}$ for the photocatalytic degradation of organic pollutants and the photocatalytic mechanism of S-ZnO is not clear.

Herein, we utilized ion exchange and calcination methods for the synthesis of S-ZnO photocatalysts, which resulted in the uniform distribution of sulfur ions in $\mathrm{ZnO}$. We discovered that the $\mathrm{S}$ element doped was mainly $\mathrm{S}^{4+}$ and $\mathrm{S}^{6+}$. The obtained $\mathrm{S}^{-}$ $\mathrm{ZnO}$ sample extended the photoresponse range for $\mathrm{ZnO}$. In order to explore $\mathrm{S}$ element doped $\mathrm{ZnO}$, the photogenerated charge properties of S-ZnO were characterized by surface photovoltage spectroscopy (SPS). In this paper, a possible photocatalytic mechanism has been proposed.

\section{Experimental section}

\section{Material synthesis}

The $\mathrm{ZnO}$ nanoflowers were synthesized using the hydrothermal method. First, $0.878 \mathrm{~g} \mathrm{Zn}\left(\mathrm{CH}_{3} \mathrm{COO}\right)_{2} \cdot 2 \mathrm{H}_{2} \mathrm{O}$ was dispersed in $60 \mathrm{~mL}$ deionized water. Then, $20 \mathrm{~mL} \mathrm{NaOH}\left(3 \mathrm{~mol} \mathrm{~L}^{-1}\right)$ was added to the above solution with stirring. The clear solution turned turbid. When $\mathrm{NaOH}$ was added in excess, the solution turned from turbid to clear. The solution was stirred for 5 
additional minutes, transferred to a $100 \mathrm{~mL}$ stainless steel autoclave, and maintained at $160{ }^{\circ} \mathrm{C}$ for $10 \mathrm{~h}$. The obtained white powder was washed with water and absolute ethanol several times and dried at $80{ }^{\circ} \mathrm{C}$ for $12 \mathrm{~h}$.

$0.2 \mathrm{~g}$ of the obtained white powder was added into a roundbottom flask. $50 \mathrm{~mL} \mathrm{Na} 2 \mathrm{~S}\left(0.5 \mathrm{~mol} \mathrm{~L}^{-1}\right)$ was added to the roundbottom flask. Then, the mixed solution was maintained at $60{ }^{\circ} \mathrm{C}$ for $4 \mathrm{~h}$ with stirring. The obtained powder was washed with deionized water and ethanol. The powder was then treated with $\mathrm{NaOH}\left(4 \mathrm{~mol} \mathrm{~L}^{-1}\right)$. The sample was heated at $600{ }^{\circ} \mathrm{C}$ for $6 \mathrm{~h}$.

\section{Material characterization}

The crystal morphologies of the samples were obtained on an XL 30 ESEM FEG field-emission scanning electron microscope (FESEM; FEI Company). The X-ray diffraction patterns were recorded on a Thermo Scientific ARL X'TRA Power Diffractometer with $\mathrm{Cu} \mathrm{K} \alpha$ radiation $\left(\lambda=1.54056 \AA\right.$ ) in the $20-70^{\circ}$ range. The X-ray photoelectron spectra (XPS) of the samples were characterized with an Escalab 250 spectrometer with monochromatized $\mathrm{Al} \mathrm{K} \alpha$. The UV-Vis-NIR spectrophotometer (Shimadzu UV-3600) was used to characterize the UV-Vis diffuse reflectance spectra (UV-Vis DRS) of the samples. The SPV of the samples was characterized with an equipment reported in our earlier study.

\section{Photocatalytic reaction}

The photocatalytic activity of the $\mathrm{Ag} / \mathrm{S}-\mathrm{ZnO}$ photocatalyst was evaluated by photodegradation of methyl orange (MO). The mixed light, provided by a $500 \mathrm{~W}$ Xenon lamp, acted as the light source. $25 \mathrm{mg}$ of photocatalyst was dispersed in a $25 \mathrm{~mL} \mathrm{MO}$ solution $\left(10 \mathrm{mg} \mathrm{L}^{-1}\right)$ by ultrasonication and stirring. In order to obtain the adsorption and desorption equilibrium between the catalyst and the dye solution, the solution was stirred in the dark for $1 \mathrm{~h}$ before illumination. Then, aliquots were taken from the suspension at $30 \mathrm{~min}$ intervals. The catalytic efficiency of the photocatalyst was evaluated using a UV-2014 ultravioletvisible spectrophotometer. The results were used to monitor absorbance changes at room temperature for the MO solution at the maximum absorption wavelength (464 nm).

\section{Results and discussion}

Fig. 1 shows the XRD patterns for $\mathrm{ZnO}$ and $\mathrm{S}-\mathrm{ZnO}$. The diffraction peaks for $\mathrm{ZnO}$ at $31.5^{\circ}, 34.2^{\circ}$, and $36.2^{\circ}$ can be indexed to the wurtzite phase of $\mathrm{ZnO}$. The ratio of the (100) diffraction peak to the (002) diffraction peak was bigger than 1 , which indicates that $\mathrm{ZnO}$ grew along the polar and the nonpolar surfaces that were exposed to the outer surface. The XRD pattern for the S-ZnO sample was similar to that of $\mathrm{ZnO}$, which indicates that $\mathrm{S}$ doping may not alter the crystal structure of $\mathrm{ZnO}$. However, the intensity of the $\mathrm{ZnO}$ diffraction peaks was weaker than that of $\mathrm{S}-\mathrm{ZnO}$ because $\mathrm{S}$ doping may have decreased the crystallinity of $\mathrm{ZnO}$.

The S-ZnO morphologies were studied using a fieldemission scanning electron microscope (FESEM). As shown in Fig. 2(a), the ZnO sample after treatment with $\mathrm{Na}_{2} \mathrm{~S}$ showed self-

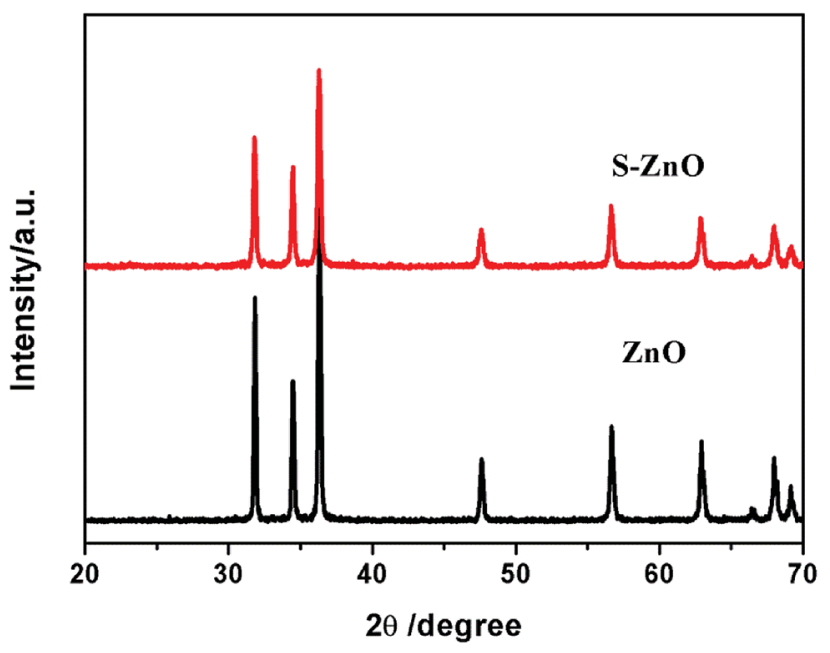

Fig. 1 The XRD patterns for $\mathrm{ZnO}$ and S-doped $\mathrm{ZnO}$.

assembled nanoflowers having nanorods with the length of 3$3.5 \mu \mathrm{m}$ and diameter of 350-700 $\mathrm{nm}$. After treatment with $\mathrm{NaOH}$, the sample showed hollow nanometer rods (shown in Fig. 2(b)). After calcination, the sample showed hollow porous nanorods, which were made of nanoparticles (shown in Fig. 2(c and $\mathrm{d})$ ).

In order to validate the valence state of $\mathrm{S}$, the $\mathrm{S}-\mathrm{ZnO}$ samples were investigated by XPS. Fig. 3 shows the XPS survey spectrum of S-ZnO, which clearly shows Zn 2p, Zn 3p, Zn 3s, C 1s, and O $1 \mathrm{~s}$ peaks. The $\mathrm{C} 1 \mathrm{~s}$ peak can be attributed to the adventitious hydrocarbon from the XPS instrument and $\mathrm{CO}_{2}$ adsorbed on the sample. ${ }^{26}$ The high-resolution S 2p XPS spectra of S-ZnO are shown in Fig. 2(b). The peaks at $169.0 \mathrm{eV}$ and $170.0 \mathrm{eV}$ can be assigned to $\mathrm{S}^{4+}$ and $\mathrm{S}^{6+}$, respectively. This result reveals that the high valent sulfur was doped into the lattice of $\mathrm{ZnO} .{ }^{27-29}$

In order to study the photoresponse properties of $\mathrm{S}-\mathrm{ZnO}$ samples, the UV-Vis diffuse reflection spectra of $\mathrm{ZnO}$ and $\mathrm{S}-\mathrm{ZnO}$ were measured. As shown in Fig. 4, the undoped $\mathrm{ZnO}$ sample showed a characteristic spectrum of pure $\mathrm{ZnO}$ with a sharp fundamental absorption edge at $\sim 390 \mathrm{~nm}$. Compared to $\mathrm{ZnO}$, the absorption range of the $\mathrm{S}-\mathrm{ZnO}$ sample showed a significant red shift to the visible light region $(550 \mathrm{~nm})$ after $S$ doping, which indicated that $\mathrm{S}-\mathrm{ZnO}$ can utilize the visible range.

In order to detect the photogenerated charge characteristics of S-ZnO, a surface photovoltage technique (SPV) was used. SPV is a powerful technique used to study the transfer behavior of photogenerated charge carriers with high sensitivity $\left(10^{8} \mathrm{q}\right.$ $\mathrm{cm}^{-2}$ ). Fig. 5(a) shows the SPV spectra of ZnO and S-ZnO. It can be seen that $\mathrm{ZnO}$ exhibits a high photovoltage response band (300-385 nm) with the response edge of $385 \mathrm{~nm}$, which is related to the intrinsic transition of $\mathrm{ZnO}$. The band gap of $\mathrm{ZnO}$ was $3.22 \mathrm{eV}$. The photovoltage response of $\mathrm{ZnO}$ in the 300$385 \mathrm{~nm}$ range was positive (shown in Fig. 5(a)) and the corresponding phase spectrum was in the range of $0-90^{\circ}$ (shown in Fig. 5(b)), which indicated that the photogenerated holes transferred to the photoelectrode under ultraviolet light. ${ }^{30,31}$ The photovoltage response range of $\mathrm{S}-\mathrm{ZnO}$ compared to that of $\mathrm{ZnO}$ extended into the visible light region $(500 \mathrm{~nm})$ with the 


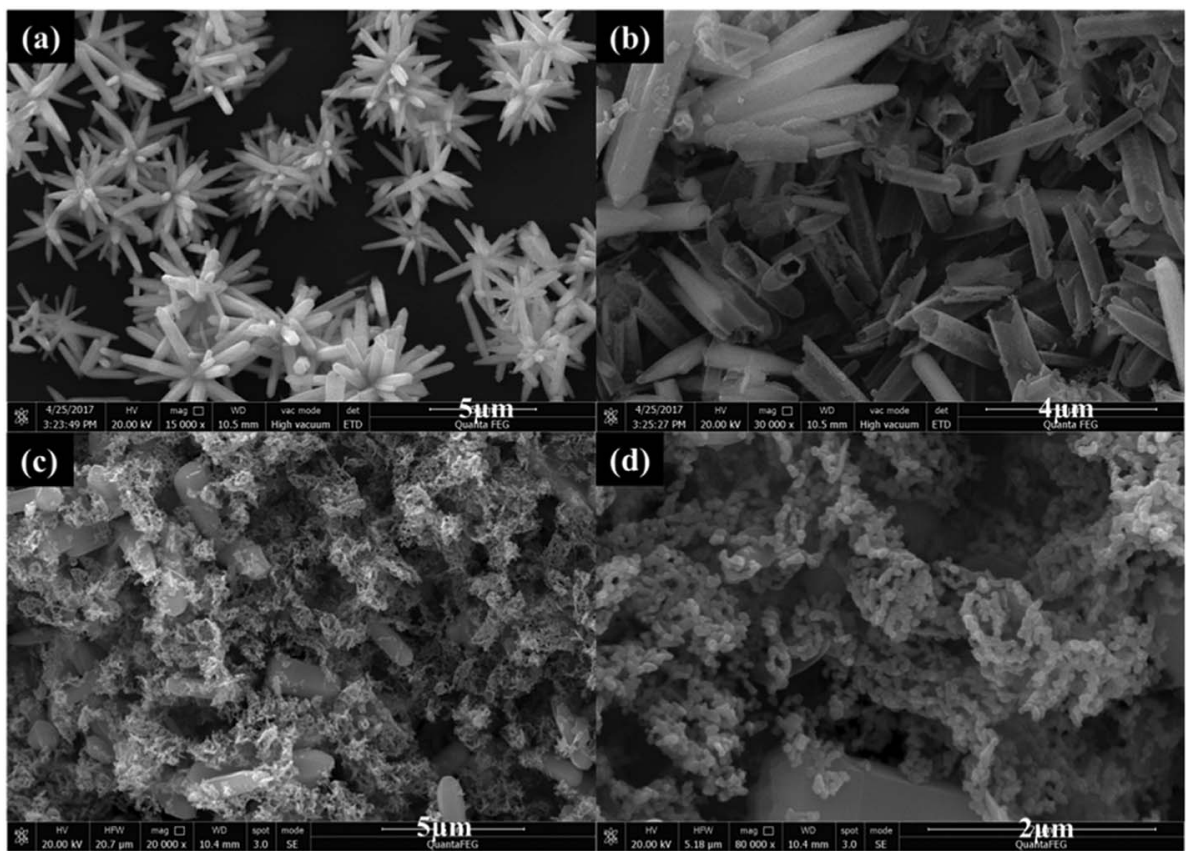

Fig. 2 FESEM images of (a) $\mathrm{ZnO}$ after treating with $\mathrm{Na}_{2} \mathrm{~S}$, (b) $\mathrm{ZnO}$ after treating with $\mathrm{NaOH}$, (c and d) $\mathrm{S}-\mathrm{ZnO}$.
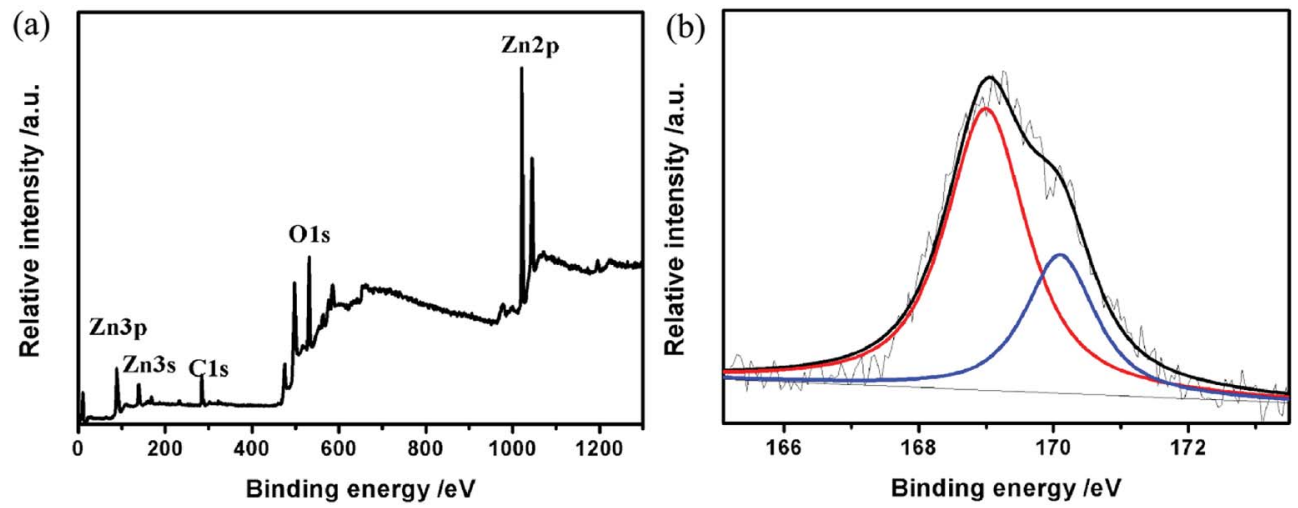

Fig. 3 XPS survey spectrum of S-ZnO (a). High-resolution S 2p XPS spectra (b).

response edge of $435 \mathrm{~nm}$, which indicated that the band gap of $\mathrm{S}-\mathrm{ZnO}$ was $2.85 \mathrm{eV}$. Yu et al. reported that the theoretical calculation of the first-principle density function showed that the ZnO band gap could be reduced with S doping. ${ }^{32}$ The S-ZnO sample was synthesized by ion exchange and calcination, which resulted in the uniform distribution of sulfur ions in $\mathrm{ZnO}$. The uniform distribution of anion dopants could elevate the valence band maximum by mixing anion doped states with the upper valence band states of the bulk material. ${ }^{33,34}$ According to the tested results of SPS (in Fig. 4(a)), the SPS response edge f S-ZnO extended to $435 \mathrm{~nm}$, which indicated that the valence band maxima of $\mathrm{S}-\mathrm{ZnO}$ was $0.37 \mathrm{eV}$ higher than that of $\mathrm{ZnO}$ whose SPS response edge was $385 \mathrm{~nm}$. According to the band-to-band transition, the photovoltage response of S-ZnO was in the range of 300-435 $\mathrm{nm}$ and the phase spectrum was in the range of $0-$ $90^{\circ}$, which indicated that the photogenerated holes transferred to the photoelectrode. The photovoltage response of S-ZnO in

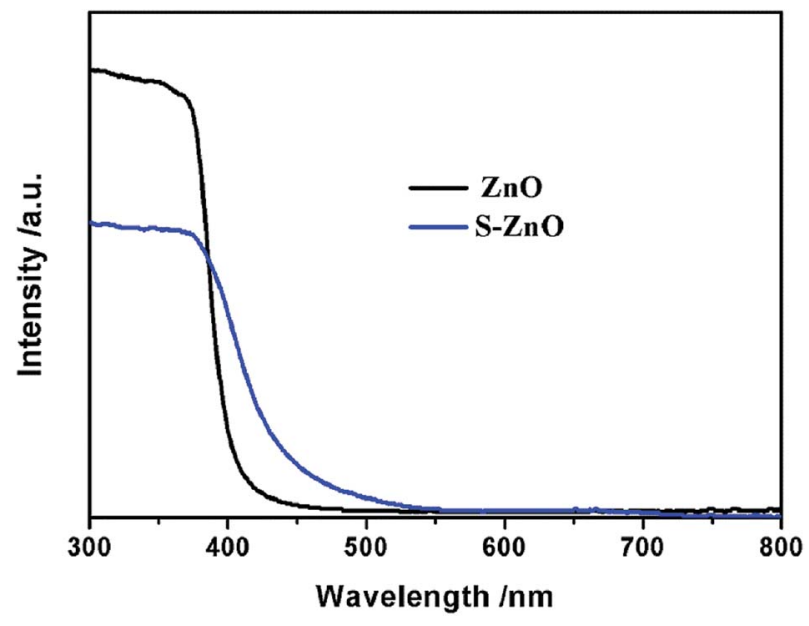

Fig. 4 The UV-Vis-NIR spectra of ZnO and S-ZnO. 

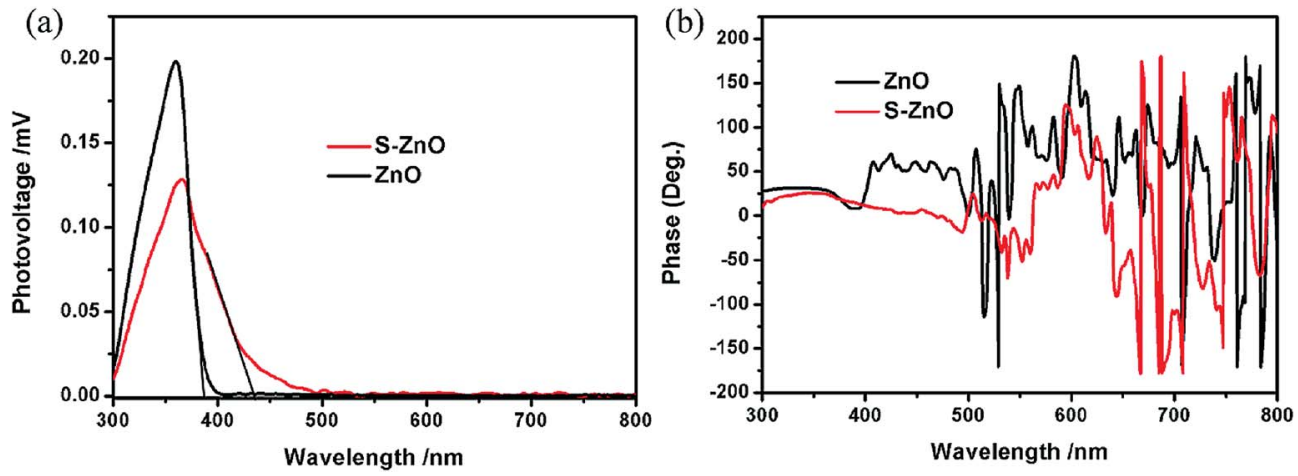

Fig. 5 The surface photovoltage spectrum (SPS) of $\mathrm{ZnO}$ and $\mathrm{S}-\mathrm{ZnO}$ (a). The phase spectra of $\mathrm{ZnO}$ and S-ZnO (b).
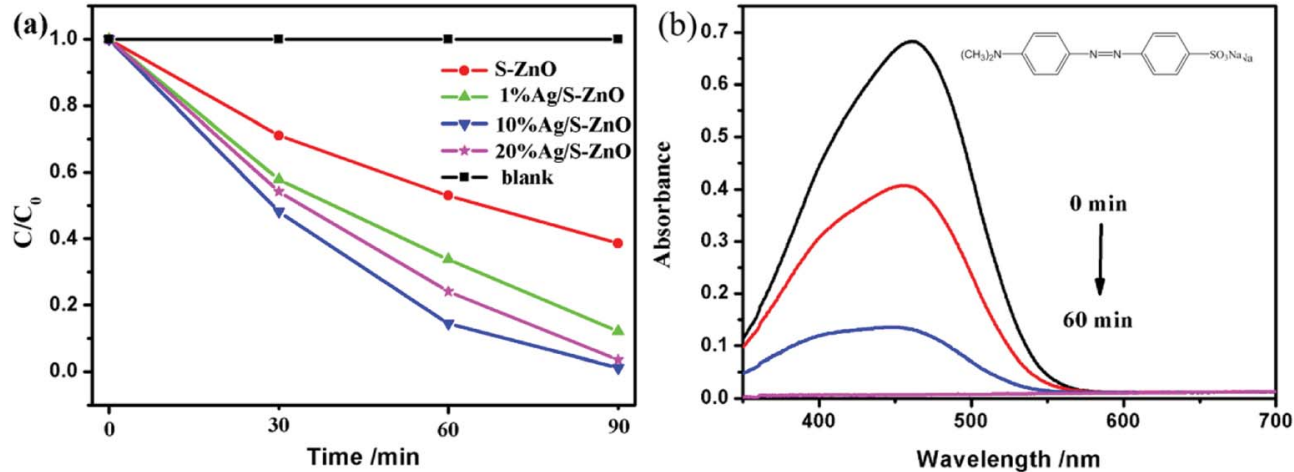

Fig. 6 Kinetics of the MO photodegradation under light illumination for Ag/S-ZnO with various Ag/S-ZnO mass fractions as catalysts (a). The absorption spectra of the photodegradation of $\mathrm{MO}$ using $\mathrm{Ag} / \mathrm{ZnO}$ sample (the inset shows the molecular structural formula of $\mathrm{MO}$ ) (b).

the range of $435-500 \mathrm{~nm}$ is due to the sub-gap transition. However, as seen in Fig. 4(a), the photovoltage response intensity of S-ZnO in the range of 300-380 $\mathrm{nm}$ was weaker than that of ZnO. This result, which is consistent with XRD analysis, may be due to the lattice defect caused by the doping of sulfur element. According to the band-to-band transition, the SPS response was not sensitive to external bias. Therefore, we tested the SPS response of S-ZnO at $410 \mathrm{~nm}$ with external bias. As shown in Fig. S1, $\dagger$ the SPS responses of S-ZnO with $0,0.1,0.5$, and $1 \mathrm{~V}$ external bias at $410 \mathrm{~nm}$ were $4.6469 \times 10^{-5}, 4.8272 \times$ $10^{-5}, 4.7222 \times 10^{-5}$, and $4.2552 \times 10^{-5} \mathrm{~V}$, respectively. This result also indicates that the SPS response of S-ZnO at $410 \mathrm{~nm}$ was based on the band-to-band transition.

In order to evaluate the photocatalytic activity of the S-ZnO sample, the degradation of a standard organic dye, methyl orange (MO), was used as a probe reaction (molecular structural formula of MO is shown in Fig. 6(b)). Fig. 6(a) shows the degradation spectra of $\mathrm{Ag} / \mathrm{S}-\mathrm{ZnO}$ with various $\mathrm{Ag} / \mathrm{S}-\mathrm{ZnO}$ mass fractions. As shown in Fig. 6(a), no degradation activity was obtained without a catalyst, which implied that the reaction was light-catalyzed. Pure S-ZnO showed poor degradation activity with light, which caused the easy recombination of the photogenerated charges in S-ZnO. After illumination for $90 \mathrm{~min}$, only about $62 \%$ of methyl orange (MO) degraded. Compared with pure $\mathrm{S}-\mathrm{ZnO}, \mathrm{Ag} / \mathrm{S}-\mathrm{ZnO}$ composites exhibited significant improvement in the photocatalytic degradation of MO. In addition, $\mathrm{Ag} / \mathrm{S}-\mathrm{ZnO}$ with $10 \mathrm{wt} \% \mathrm{Ag}$ loading showed the highest photocatalytic degradation rate for MO. After illumination for $90 \mathrm{~min}$, about $100 \%$ of methyl orange (MO) was degraded.

Based on the above analysis, we proposed a possible photocatalytic mechanism. The potential photogenerated charge transfer process of $\mathrm{S}-\mathrm{ZnO}$ is shown in Scheme 1. The uniform

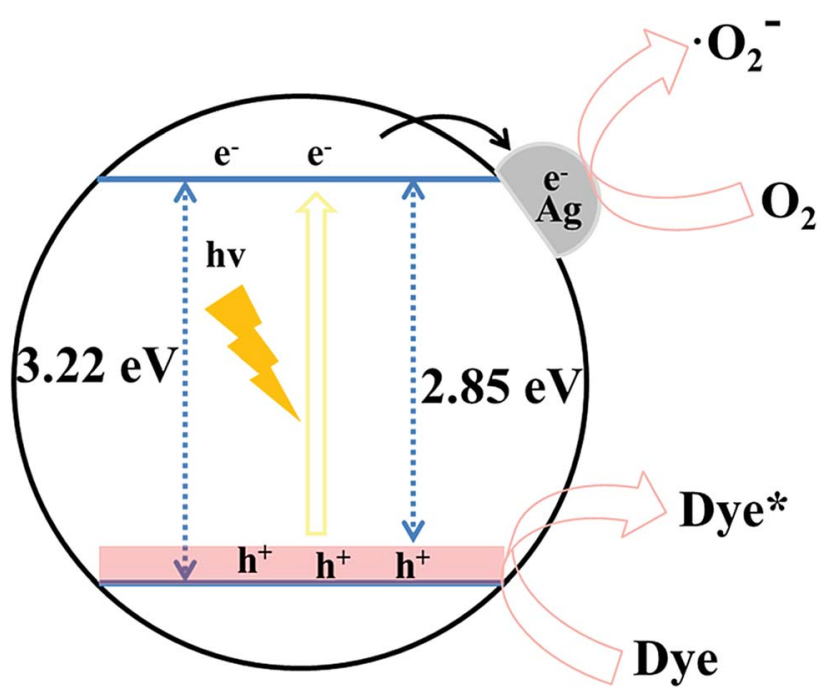

Scheme 1 The schematic illustration of the photocatalytic mechanism of $\mathrm{S}-\mathrm{ZnO}$. 
distribution of $\mathrm{S}$ dopants could elevate the valence band maximum by mixing $S 3$ with the upper valence band states of $\mathrm{ZnO}$. The valence band maxima of $\mathrm{S}-\mathrm{ZnO}$ was $0.37 \mathrm{eV}$ higher than that of $\mathrm{ZnO}$. When the photogenerated electrons from the valence band of $\mathrm{S}-\mathrm{ZnO}$ were transferred to the conduction band under light, the photogenerated electrons were captured by Ag. This resulted in the formation of a superoxide radical with oxygen molecules on the surface. The photogenerated holes in the valence band of S-ZnO degraded MO.

\section{Conclusion}

In this study, the ZnO doped with a high valent S element was successfully prepared by ion exchange and calcination methods, resulting in the uniform distribution of sulfur ions in $\mathrm{ZnO}$. We studied the influence of the uniform distribution of $\mathrm{S}$ $\left(\mathrm{S}^{4+}\right.$ and $\left.\mathrm{S}^{6+}\right)$ doping on photogenerated charge characteristics of $\mathrm{ZnO}$ with SPS. The photoelectron response of $\mathrm{ZnO}$ was extended to $500 \mathrm{~nm}$ due to the high valent $\mathrm{S}$ doping. The uniform distribution of $\mathrm{S}$ dopants elevated the valence band maximum by mixing $S 3$ p with the upper valence band states of $\mathrm{ZnO}$. The valence band maxima of $\mathrm{S}-\mathrm{ZnO}$ was $0.37 \mathrm{eV}$ higher than that of $\mathrm{ZnO}$. Ag/S-ZnO, with $10 \mathrm{wt} \% \mathrm{Ag}$ loading, showed the highest photocatalytic degradation rate for MO.

\section{Conflicts of interest}

There are no conflicts to declare.

\section{Acknowledgements}

For financial support, we are grateful to the National Natural Science Foundation of China (No. 51574130), the National Science Youth Foundation of China (No. 51704123, No. 21602074, and No. 21605055), the Foundation of Key Laboratory for Palygorskite Science and Applied Technology of Jiangsu Province (No. HPK201504) and the Provincial Science Youth Foundation of Jiangsu (No. BK20160424, No. BK20160425, and No. BK20150416).

\section{References}

1 S. Rajendran, M. M. Khan, F. Gracia, et al., $\mathrm{Ce}^{3+}$-ion-induced visible-light photocatalytic degradation and electrochemical activity of $\mathrm{ZnO} / \mathrm{CeO}_{2}$ nanocomposite, Sci. Rep., 2016, 6, 31641.

2 P. K. Sanoop, S. Anas, S. Ananthakumar, et al., Synthesis of yttrium doped nanocrystalline $\mathrm{ZnO}$ and its photocatalytic activity in methylene blue degradation, Arabian J. Chem., 2016, 9, S1618-S1626.

$3 \mathrm{X}$. Chen, Z. Wu, D. Liu, et al., Preparation of $\mathrm{ZnO}$ Photocatalyst for the Efficient and Rapid Photocatalytic Degradation of Azo Dyes, Nanoscale Res. Lett., 2017, 12(1), 143.

$4 \mathrm{H}$. Sudrajat and S. Babel, Comparison and mechanism of photocatalytic activities of $\mathrm{N}-\mathrm{ZnO}$ and $\mathrm{N}-\mathrm{ZrO} 2$ for the degradation of rhodamine 6G, Environ. Sci. Pollut. Res., 2016, 23(10), 10177-10188.

$5 \mathrm{H}$. Sudrajat and S. Babel, Comparison and mechanism of photocatalytic activities of $\mathrm{N}-\mathrm{ZnO}$ and $\mathrm{N}-\mathrm{ZrO}_{2}$ for the degradation of rhodamine 6G, Environ. Sci. Pollut. Res., 2016, 23(10), 10177-10188.

6 Y. Yuan, G. F. Huang, W. Y. Hu, et al., Tunable synthesis of various $\mathrm{ZnO}$ architectural structures with enhanced photocatalytic activities, Mater. Lett., 2016, 175, 68-71.

7 Y. M. Hunge, A. A. Yadav, S. B. Kulkarni, et al., A multifunctional $\mathrm{ZnO}$ thin film based devices for photoelectrocatalytic degradation of terephthalic acid and $\mathrm{CO}_{2}$ gas sensing applications, Sens. Actuators, B, 2018, 274, 1-9.

8 T. Xu, L. Zhang, H. Cheng, et al., Significantly enhanced photocatalytic performance of $\mathrm{ZnO}$ via graphene hybridization and the mechanism study, Appl. Catal., B, 2011, 101(3-4), 382-387.

9 Y. Wang, R. Shi, J. Lin, et al., Enhancement of photocurrent and photocatalytic activity of $\mathrm{ZnO}$ hybridized with graphitelike $\mathrm{C}_{3} \mathrm{~N}_{4}$, Energy Environ. Sci., 2011, 4(8), 2922-2929.

10 Y. Yuan, G. F. Huang, W. Y. Hu, et al., Construction of g$\mathrm{C}_{3} \mathrm{~N}_{4} / \mathrm{CeO}_{2} / \mathrm{ZnO}$ ternary photocatalysts with enhanced photocatalytic performance, J. Phys. Chem. Solids, 2017, 106, 1-9.

11 J. H. Xiao, W. Q. Huang, Y. Hu, et al., Facile in situ synthesis of wurtzite $\mathrm{ZnS} / \mathrm{ZnO}$ core/shell heterostructure with highly efficient visible-light photocatalytic activity and photostability, J. Phys. D: Appl. Phys., 2018, 51(7), 075501.

12 N. Li, Y. Tian, J. Zhao, et al., Z-scheme 2D/3D g- $\mathrm{C}_{3} \mathrm{~N}_{4}$ @ ZnO with enhanced photocatalytic activity for cephalexin oxidation under solar light, Chem. Eng. J., 2018, 352, 412422.

13 Y. Zheng, L. Zheng, Y. Zhan, et al., Ag/ZnO heterostructure nanocrystals: synthesis, characterization, and photocatalysis, Inorg. Chem., 2007, 46(17), 6980-6986.

14 G. Poongodi, P. Anandan, R. M. Kumar, et al., Studies on visible light photocatalytic and antibacterial activities of nanostructured cobalt doped $\mathrm{ZnO}$ thin films prepared by sol-gel spin coating method, Spectrochim. Acta, Part A, 2015, 148, 237-243.

15 Y. G. Habba, M. Capochichi-Gnambodoe and Y. LeprinceWang, Enhanced Photocatalytic Activity of Iron-Doped ZnO Nanowires for Water Purification, Appl. Sci., 2017, 7(11), 1185.

16 W. Choi, A. Termin and M. R. Hoffmann, The Role of Metal Ion Dopants in Quantum-Sized $\mathrm{TiO}_{2}$ : Correlation between Photoreactivity and Charge Carrier Recombination Dynamics, J. Phys. Chem., 1994, 98(51), 13669-13679.

17 R. Asahi, T. Morikawa, T. Ohwaki, et al., Visible-light photocatalysis in nitrogen-doped titanium oxides, Science, 2001, 293(5528), 269-271.

18 Z. Yu, L. C. Yin, Y. Xie, et al., Crystallinity-dependent substitutional nitrogen doping in $\mathrm{ZnO}$ and its improved visible light photocatalytic activity, J. Colloid Interface Sci., 2013, 400(12), 18-23.

19 Y. Qiu, K. Yan, H. Deng, et al., Secondary branching and nitrogen doping of $\mathrm{ZnO}$ nanotetrapods: building a highly 
active network for photoelectrochemical water splitting, Nano Lett., 2012, 12(1), 407.

20 S. H. Choi, D. Lim, J. W. Park, et al., The Role of Carbon Doping in ZnO, J. Korean Phys. Soc., 2010, 57(6), 1482-1485.

21 P. M. R. Kumar, C. S. Kartha, K. P. Vijayakumar, et al., Effect of fluorine doping on structural, electrical and optical properties of ZnO thin films, Mater. Sci. Eng., B, 2005, 117(3), 307-312.

22 H. Qin, W. Li, Y. Xia, et al., Photocatalytic activity of heterostructures based on $\mathrm{ZnO}$ and N-doped $\mathrm{ZnO}$, ACS Appl. Mater. Interfaces, 2011, 3(8), 3152-3156.

$23 \mathrm{D}$. Li and H. Haneda, Synthesis of nitrogen-containing $\mathrm{ZnO}$ powders by spray pyrolysis and their visible-light photocatalysis in gas-phase acetaldehyde decomposition, $J$. Photochem. Photobiol., A, 2003, 155(1-3), 171-178.

24 L. L. Feng, Y. Zou, C. Li, et al., Nanoporous sulfur-doped graphitic carbon nitride microrods: A durable catalyst for visible-light-driven $\mathrm{H}_{2}$ evolution, Int. J. Hydrogen Energy, 2014, 39(28), 15373-15379.

25 Q. Xiang, J. Yu and M. Jaroniec, Nitrogen and sulfur codoped $\mathrm{TiO}_{2}$ nanosheets with exposed $\{001\}$ facets: synthesis, characterization and visible-light photocatalytic activity, Phys. Chem. Chem. Phys., 2011, 13(11), 4853-4861.

$26 \mathrm{~J} . \mathrm{Yu}, \mathrm{Y}$. Hai and B. Cheng, Enhanced photocatalytic $\mathrm{H}_{2}$ production activity of $\mathrm{TiO}_{2}$ by $\mathrm{Ni}(\mathrm{OH})_{2}$ cluster modification, J. Phys. Chem. C, 2011, 115, 4953-4958.

27 T. Ohno, T. Tsubota, Y. Nakamura and K. Sayama, Preparation of $\mathrm{S}, \mathrm{C}$ cation-codoped $\mathrm{SrTiO}_{3}$ and its photocatalytic activity under visible light, Appl. Catal., A, 2005, 288, 74-79.

28 T. Ohno, T. Mitsui and M. Matsumura, Photocatalytic activity of S-doped $\mathrm{TiO}_{2}$ photocatalyst under visible light, Chem. Lett., 2003, 32, 364-365.

29 J. C. Yu, W. Ho, J. G. Yu, H. Yip, P. K. Wong and J. C. Zhao, Efficient visible-light-induced photocatalytic disinfection on sulfur-doped nanocrystalline titania, Environ. Sci. Technol., 2005, 39, 1175-1179.

30 H. Fan, T. Jiang, H. Li, et al., Effect of BivO4 Crystalline Phases on the Photoinduced Carriers Behavior and Photocatalytic Activity, J. Phys. Chem. C, 2016, 116(3), 24252430.

31 T. Jiang, T. Xie, W. Yang, et al., Photoelectrochemical and Photovoltaic Properties of $\mathrm{p}-\mathrm{n} \mathrm{Cu}_{2} \mathrm{O}$ Homojunction Films and Their Photocatalytic Performance, J. Phys. Chem. C, 2013, 117(9), 4619-4624.

$32 \mathrm{~W}$. Yu, J. Zhang and T. Peng, New insight into the enhanced photocatalytic activity of $\mathrm{N}$-, C-and S-doped $\mathrm{ZnO}$ photocatalysts, Appl. Catal., B, 2016, 181, 220-227.

33 G. Liu, L. Wang, H. G. Yang, et al., Titania-based photocatalysts-crystal growth, doping and heterostructuring, J. Mater. Chem., 2010, 20(5), 831-843.

34 G. Liu, P. Niu, C. Sun, et al., Unique electronic structure induced high photoreactivity of sulfur-doped graphitic $\mathrm{C}_{3} \mathrm{~N}_{4}$, J. Am. Chem. Soc., 2010, 132(33), 11642-11648. 